Pacific

Journal of

Mathematics

\title{
RINGS WITH SOVABLE QUIVERS
}

Frank W. Anderson and Barbara K. D'Ambrosia

Volume $213 \quad$ No. 2

February 2004 


\title{
RINGS WITH SOVABLE QUIVERS
}

\author{
Frank W. Anderson and Barbara K. D'Ambrosia
}

\begin{abstract}
An artinian ring $R$ is square-free in case none of its indecomposable projective modules has a repeated composition factor. Let $\mathcal{Q}$ be the quiver of such a square-free ring $R$. In this paper we show that if $R$ is indecomposable and transitive on the cyclic components of $\mathcal{Q}$ and if $\mathcal{Q}$ contains no $n$-crown, then $R \cong D \otimes_{K} A$ where $D$ is the natural division ring of $R, K=$ Cen $D$, and $A$ is a square-free $K$-algebra; that is, $\operatorname{dim}_{K}(e A f) \leq 1$ for every pair $e, f \in A$ of primitive idempotents.
\end{abstract}

\section{Introduction.}

Let $R$ be an artinian ring with basic $\operatorname{ring} \bar{R}$, and suppose that $\bar{R} / \bar{J} \cong D^{n}$ (as rings) for some division ring $D$ and some $n \in \mathbb{N}$. Then $R$ is said to be split with division ring $D$. If $K=\operatorname{Cen} D$, then $R$ is a $D$-algebra in case also $R \cong D \otimes_{K} A$ for some (necessarily split) $K$-algebra $A$. Much of the study of such a $D$-algebra reduces to that of the usually more tractable $K$-algebra $A$. Of course, it is not common for an artinian ring $R$ to be split and even less common for it to be a $D$-algebra. Nevertheless, it is of some interest to identify classes of artinian rings that can be realized as $D$-algebras. Fortunately, as we show in Theorem 3.2, the property of being a $D$-algebra is Morita invariant, and so we may focus on basic rings.

An artinian ring $R$ is square-free in case no indecomposable projective $R$ module has a repeated composition factor. In [6] D'Ambrosia has shown that every indecomposable square-free ring is split. Moreover, there it is shown that there exist substantial classes of indecomposable square-free rings that are not $D$-algebras for any division ring $D$. In this paper we find a large class of square-free rings that are necessarily $D$-algebras.

Each artinian ring $R$ determines two digraphs, the quivers of $R$. (See, for example, [7].) If $R$ is square-free, then these two quivers are order-theoretic duals (see [6] Lemma $2.1^{1}$ ), so we work with the right quiver $\mathcal{Q}$ of $R$. We say that $\mathcal{Q}$ is solvable if it contains no $n$-crown (see Section 2 ). So, for

\footnotetext{
${ }^{1}$ In [6], D'Ambrosia inadvertently reversed the roles of left and right quivers. The reader should replace "left quiver" with "right quiver" and vice versa when referring to that paper.
} 
example, if $\mathcal{Q}$ is a tree or has a unique source or a unique $\operatorname{sink}$, then $\mathcal{Q}$ is solvable.

Fuller and Haack ([7]) studied rings $R$ whose quivers are trees. Such rings are necessarily square-free and solvable. The main result of $[\mathbf{7}]$ is that, in effect, if the quivers of $R$ are trees, then $R$ is a $D$-algebra and its basic ring is isomorphic to a factor ring of the $D$-incidence ring of a poset.

In this paper we consider the more general square-free rings with solvable quivers. Let $\mathcal{Q}$ be the quiver of a square-free ring $R$. If all cut edges of $\mathcal{Q}$, viewed as an undirected graph, are removed, the connected components of the resulting graph are the cyclic components of $\mathcal{Q}$. For the solvability of $\mathcal{Q}$ to be reflected in the behavior of $R$ we need that $R$ be transitive on each of these cyclic components; we say then that $R$ is locally transitive. (See Section 4.) So if $\mathcal{Q}$ is a tree, then it is locally transitive. Our main result, Theorem 4.8, is that if $R$ is an indecomposable, locally transitive, square-free ring with solvable quiver, then $R$ is a $D$-algebra.

Finally, in Section 5 we look at a generalization of Gabriel's Theorem for split $K$-algebras. We show that a $D$-algebra is a factor of the $D$-path algebra of its quiver. From this it follows that, if $R$ is basic square-free locally transitive with solvable quiver, then $R$ is a twisted factor ring of the $D$-incidence algebra of its quiver.

\section{Solvable quivers.}

By a quiver we will mean a finite digraph $\mathcal{Q}=(V, A)$ with vertex set $V$ and arrow set $A$. By a $d$-path (or directed path) in $\mathcal{Q}$ we mean a sequence of arrows

$$
p=\left(a_{1}, a_{2}, \ldots, a_{m}\right)
$$

such that for each $1 \leq i<m$ the terminal vertex of $a_{i}$ is the initial vertex of $a_{i+1}$. A $d$-path with $m$ arrows has length $m$. A single vertex $v$ will be considered as a degenerate (trivial) $d$-path of length 0 with initial and terminal vertices both $v$. A bi-directed cycle in $\mathcal{Q}$ is the union $p \cup q$ of two $d$-paths of $\mathcal{Q}$ that intersect only in a common initial vertex and common terminal vertex.

We will also want to view the pair $(V, A)$ as an undirected graph; that is, in this context we consider each $a \in A$ as an unoriented edge. Then by a path in $\mathcal{Q}$ we mean a path in the undirected graph $(V, A)$. A cycle in $\mathcal{Q}$ is just a nontrivial path that is simply closed. Thus, every $d$-path of $\mathcal{Q}$ is a path of $\mathcal{Q}$, and every bi-directed cycle of $\mathcal{Q}$ is a cycle of $\mathcal{Q}$ or a union of cycles such as two loops with the same vertex. But the converses of these statements are true only in degenerate examples. 
Example 2.1. Consider the following quivers:

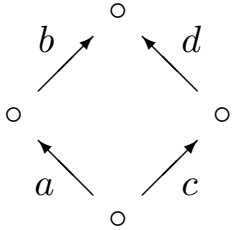

$\mathcal{Q}_{1}$

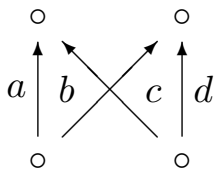

$\mathcal{Q}_{2}$

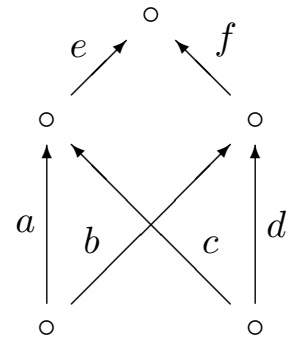

$\mathcal{Q}_{3}$

Here $\mathcal{Q}_{1}$ has one cycle and one bi-directed cycle, the union of the two $d$-paths $(a, b)$ and $(c, d)$. The quiver $\mathcal{Q}_{2}$ has one cycle and no bi-directed cycles. The graph $\mathcal{Q}_{3}$ has two bi-directed cycles and one other cycle.

Let $\mathcal{Q}=(V, A)$ be a quiver that contains no directed cycles and hence no loops. A vertex is a source if it is the head of no arrow and it is a sink if it is the tail of no arrow. Since our quiver is finite and contains no directed cycles, it must have at least one source and at least one sink. Let $U=\left\{u_{1}, \ldots, u_{n}\right\}$ be a set of sources and $W=\left\{w_{1}, \ldots, w_{n}\right\}$ a set of sinks in $\mathcal{Q}$. If $n \geq 2$, then $U$ and $W$ determine an $n$-crown if:

(C1) There is a $d$ path from $u_{i}$ to $w_{j}$ iff $i>1$ and $i-1 \leq j \leq i$, or $(i, j)=(1,1)$, or $(i, j)=(1, n)$.

(C2) If $n=2$, no path from $u_{1}$ to $w_{2}$ shares a vertex with any path starting at $u_{2}$ and ending at $w_{1}$.

So, for example, the quiver $\mathcal{Q}_{2}$ of Example 2.1 is a 2-crown. For $n>2$ an $n$-crown has the form

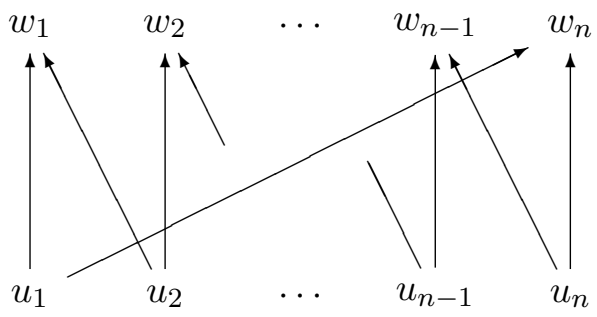

where the arrows indicate directed paths of unspecified length. We say the quiver $\mathcal{Q}$ is solvable in case it contains no $n$-crown. So note that if $\mathcal{Q}$ has either a single source or a single sink, then $\mathcal{Q}$ is solvable.

Let $U=\left\{u_{1}, \ldots, u_{n}\right\}$ be a set of sources and $W=\left\{w_{1}, \ldots, w_{n}\right\}$ a set of sinks in $\mathcal{Q}$. If there are $d$-paths from $u_{i}$ to $w_{i}$ and $w_{i-1}$ for all $1<i \leq n$ and from $u_{1}$ to $w_{1}$ and to $w_{n}$, then we say that $U$ and $W$ determine a weak $n$-crown. So an $n$-crown is a weak $n$-crown but not conversely. 
Lemma 2.2. Suppose that $\mathcal{Q}$ is a connected solvable quiver. Let $U=$ $\left\{u_{1}, \ldots, u_{n}\right\}$ be a set of sources and $W=\left\{w_{1}, \ldots, w_{n}\right\}$ a set of sinks in $\mathcal{Q}$. If $U$ and $W$ determine a weak $n$-crown, then there exist $1<i \leq n$, $1 \leq j<n$ and a vertex $v$ with d-paths from $u_{1}$ to $w_{n}$ through $v$ and from $u_{i}$ to $w_{j}$ through $v$.

Proof. We'll prove this by induction. For $n=2$ this immediate from (C2). So let $n>2$. Since $\mathcal{Q}$ has no $n$-crowns, there is a $d$-path from some $u_{i}$ to some $w_{j}$ where

$$
0<j-i<n-1 \quad \text { or } \quad 1<i-j<n .
$$

In the first case

$$
U^{\prime}=\left\{u_{1}, \ldots, u_{i}, u_{j+1} \ldots, u_{n}\right\} \quad \text { and } \quad W^{\prime}=\left\{w_{1}, \ldots, w_{i-1}, w_{j}, \ldots, w_{n}\right\}
$$

form a weak $n-j+i$-crown. In the second,

$$
U^{\prime}=\left\{u_{1}, \ldots, u_{j}, u_{i}, \ldots, u_{n}\right\} \quad \text { and } \quad W^{\prime}=\left\{w_{1}, \ldots, w_{j}, w_{i}, \ldots, w_{n}\right\}
$$

form a weak $n-i+j+1$-crown. So by induction there exist the desired vertex $v$ and $d$-paths.

Now let $\mathcal{Q}=(V, A)$ be a quiver. By a subquiver of a quiver $\mathcal{Q}$ we mean a quiver whose vertex and arrow sets are subsets of $V$ and $A$. If $H \subseteq A$, then we may view $H$ as a subquiver with arrow set $H$. If $H$ and $K$ are two subquivers of $\mathcal{Q}$, then $H \cup K$ is the subquiver whose vertex and arrow sets are the unions of those of $H$ and $K$, and $H \cap K$ is the subquiver whose vertex and arrow sets are the intersections of those of $H$ and $K$. Also, if $H \subseteq A$, then the subgraph generated by $H$ is the (unoriented) subgraph of $\mathcal{Q}$ consisting of all edges from $H$ together with the vertices of these edges. The complement of $H$ is the subgraph of $\mathcal{Q}$ generated by the edges of $\mathcal{Q}$ not in $H$.

Let $T$ be the subgraph generated by the set of all cut edges of $\mathcal{Q}$. (A cut edge is an edge whose deletion separates the graph.) Let $S$ be the complement of $T$, so each edge of $S$ belongs to at least one cycle. Let $S_{1}, \ldots, S_{r}$ be the connected components of $S$; we call these the cyclic components of $\mathcal{Q}$. The subgraph $T$ consists of a finite set of disjoint trees $T_{1}, \ldots, T_{q}$. Since each edge of $T$ is a cut edge of $\mathcal{Q}$, each tree $T_{i}$ meets any one cyclic component $S_{j}$ in at most one common vertex. And if $\mathcal{Q}$ is connected with $r \geq 1$, each tree $T_{i}$ meets at least one $S_{j}$ in a common vertex. Note that if $\mathcal{Q}$ is connected and we shrink each cyclic component $S_{i}$ to a single vertex, then the resulting graph will be a tree.

Suppose that $\mathcal{Q}$ is a finite quiver with no directed cycles. The addition or removal of a single arrow that is a cut edge of the graph will not affect the existence of an $n$-crown. Thus, we see that $\mathcal{Q}$ is solvable iff each of its cyclic components is solvable. 
For every cyclic component $S_{i}$, its star is the subgraph $\operatorname{St}\left(S_{i}\right)$ of $\mathcal{Q}$ generated by $S_{i}$ and all trees $T_{j}$ in $T$ that meet $S_{i}$. A labeling $\left\{S_{1}, \ldots, S_{r}\right\}$ of the components of $S$ is a proper order in case for each $1 \leq k \leq r$ the graph

$$
\operatorname{St}\left(S_{1}\right) \cup \operatorname{St}\left(S_{2}\right) \cup \cdots \cup \operatorname{St}\left(S_{k}\right)
$$

is connected.

Lemma 2.3. If $\mathcal{Q}$ is a connected quiver, then $\mathcal{Q}$ is a tree or there exists a proper order for the cyclic components of $\mathcal{Q}$.

Proof. Assume that $\mathcal{Q}$ is not a tree. Let $r$ be the number of cyclic components $S_{1}, \ldots, S_{r}$, and let $1 \leq k \leq r$. We induct on $k$. If $k=1$, then the result is trivial. So let $1 \leq k<r$, and assume that cyclic components $S_{1}, \ldots, S_{k}$ have been chosen so that $H=\operatorname{St}\left(S_{1}\right) \cup \operatorname{St}\left(S_{2}\right) \cup \cdots \cup \operatorname{St}\left(S_{k}\right)$ is connected. Since $\mathcal{Q}$ is connected, the complement of $H$ must have at least one vertex in common with $H$. If some tree $T_{j}$ has a vertex in common with $H$, then it lies in $H$. Thus, there must be some cyclic component $S_{k+1}$ sharing a vertex with $H$. So, clearly, $H \cup \operatorname{St}\left(S_{k+1}\right)$ is connected.

\section{Square-free $D$-algebras.}

Let $R$ be an artinian ring with radical $J$ and with basic set $E=\left\{e_{1}, \ldots, e_{n}\right\}$; that is, the set $\left\{e_{1}, \ldots, e_{n}\right\}$ consists of pairwise orthogonal primitive idempotents, and $R e_{1}, \ldots, R e_{n}$ is a complete irredundant set of the indecomposable projective left $R$-modules. (See [3], page 305.) Then (see [3], Exercise 32.14, or [7]) the (right) quiver of $R$ is the digraph $\mathcal{Q}(R)$ with vertex set $E$ and with $m_{i j}$ arrows from $e_{i}$ to $e_{j}$ where $m_{i j}$ is the multiplicity of the simple module $\overline{e_{j} R}=e_{j} R / e_{j} J$ in the semisimple module $e_{i} J / e_{i} J^{2}$. The left quiver of $R$ is defined similarly.

An artinian ring $R$ is square-free in case for every primitive idempotent $e \in R$, the indecomposable projective modules $R e$ and $e R$ have no repeated composition factors. (See [6] for the basic facts about these rings.) If $R$ is square-free, then ([6], Lemma 2.1) the left quiver of $R$ is just the ordertheoretic dual, $\mathcal{Q}(R)^{\mathrm{op}}$, of the right quiver $\mathcal{Q}(R)$. So, in particular, for a square-free ring the left quiver is solvable iff the right quiver is solvable. For the remainder of this paper we will assume that $R$ is square-free. Note that this means that the quiver $\mathcal{Q}$ of $R$ has no multiple arrows, and no arrow from a vertex to itself.

For a special case of such rings, let $A$ be a split finite dimensional algebra over a field $K$. Then the $K$-algebra $A$ is square free iff for each pair $e, f \in A$ of primitive idempotents

$$
\operatorname{dim}_{K}(e A f) \leq 1 .
$$

These algebras have been described in detail in [2]. In particular, every incidence algebra of a pre-ordered set is square-free. Observe that if $A$ is a 
square-free $K$-algebra and if $D$ is a division ring with Cen $D=K$, then

$$
R=D \otimes_{K} A
$$

is a square-free ring. A square-free ring of this form is said to be a squarefree $D$-algebra. As is shown in $[6]$, there are plenty of square-free rings that are not $D$-algebras. Let $R$ be an indecomposable square-free ring. Our main goal in this paper is to show that if $R$ is locally transitive square-free (see Section 4) with solvable quiver, then it must be a $D$-algebra for some $D$. Our intermediate goal in this section is to obtain a characterization of square-free $D$-algebras that will provide the machinery to prove the main result.

So now let $R$ be an indecomposable square-free ring. Then (see [6], Theorem 1.4) there is a division ring $D$, called the division ring of $R$, such that for every primitive idempotent $e \in R, D \cong e R e$. Moreover, for each pair $e, f \in R$ of primitive idempotents, $\operatorname{dim}_{e R e}(e R f) \leq 1$ and $\operatorname{dim}_{f R f}(e R f) \leq 1$. (See [6], Theorem 1.4.) It follows ([6], Corollary 1.5) that if $e, f \in R$ are primitive idempotents with $e R f \neq 0$, then for each $0 \neq e r f \in e R f$, there is an isomorphism

$$
\varphi_{\text {erf }}: e R e \longrightarrow f R f,
$$

that we treat as a right operator ${ }^{2}$ where $(e x e) \varphi_{\text {erf }}$ is the unique element of $f R f$ such that

$$
e x e \cdot e r f=e r f \cdot(e x e) \varphi_{e r f} .
$$

This is the inverse of the isomorphism given in [6]. Concerning the isomorphisms $\varphi_{\text {erf }}$, we will use the following slight variation of Corollary 1.5 from $[6]$ :

Lemma 3.1. Let e, $f, g \in R$ be primitive idempotents in the square-free ring $R$ and let $r, s, t \in R$. If erfsg $=($ eae $)$ etg $\neq 0$ for some eae $\in \operatorname{Cen}(e R e)$, then

$$
\varphi_{e r f} \varphi_{f s g}=\varphi_{e t g}
$$

Proof. For each exe $\in$ eRe,

$$
\begin{aligned}
(\text { etg }) \cdot(\text { exe }) \varphi_{e r f} \varphi_{f s g} & =(\text { eae })^{-1} \text { erfsg } \cdot(\text { exe }) \varphi_{\text {erf }} \varphi_{f s g} \\
& =(\text { eae })^{-1} \mathrm{erf} \cdot(\text { exe }) \varphi_{e r f} \cdot f s g \\
& =(\text { eae })^{-1} \cdot \text { exe } \cdot \mathrm{erf} s g \\
& =(\text { exe })(\text { eae })^{-1} \mathrm{erfsg} \\
& =(\text { exe })(\text { etg }) \\
& =(\text { etg })(\text { exe }) \varphi_{\text {etg }} .
\end{aligned}
$$

And so $\varphi_{e r f} \varphi_{f s g}=\varphi_{e t g}$.

\footnotetext{
${ }^{2}$ In this section and the next all maps will be written as right operators unless explicitly stated otherwise.
} 
Next, we observe that the property of being a $D$-algebra is Morita invariant, so that we can reduce to the basic case.

Theorem 3.2. Let $R$ and $S$ be Morita equivalent rings. If $R \cong D \otimes_{K} A$ is a D-algebra, then there is a $K$-algebra $A^{\prime}$, Morita equivalent to $A$, such that $S \cong D \otimes_{K} A^{\prime}$.

Proof. Suppose that $R=D \otimes_{K} A$. If $e, f \in A$ are primitive idempotents with $A e \cong A f$, then $1 \otimes e$ and $1 \otimes f$ are primitive idempotents of $R$ that satisfy $R(1 \otimes e) \cong R(1 \otimes f)$. Thus, $\left\{e_{1}, \ldots, e_{n}\right\}$ is a basic set of primitive idempotents for $A$ iff $\left\{1 \otimes e_{1}, \ldots, 1 \otimes e_{n}\right\}$ is a basic set for $R$. Assuming that those sets are basic, then

$$
P^{\prime}=\bigoplus_{i=1}^{n}\left(R\left(1 \otimes e_{i}\right)\right)^{m_{i}}=D \otimes_{K} \bigoplus_{i=1}^{n}\left(A e_{i}\right)^{m_{i}}
$$

is a progenerator for $R$ iff $m_{i} \geq 1$ for each $i$, iff $P=\oplus_{i=1}^{n}\left(A e_{i}\right)^{m_{i}}$ is a progenerator for $A$. So it will suffice to prove that if ${ }_{A} P$ is a finitely generated projective $A$-module, then (see $[\mathbf{3}]$, Corollary 22.4)

$$
\operatorname{End}_{R}\left(D \otimes_{K} P\right) \cong D \otimes_{K} \operatorname{End}_{A}(P) .
$$

For in this case, we let $A^{\prime}=\operatorname{End}_{A}(P)$, where $P$ is a progenerator for $A$ as described above.

But if $P=A^{n}$ is free, then

$$
\begin{aligned}
\operatorname{End}_{R}\left(D \otimes_{K} A^{n}\right) & \cong \operatorname{End}_{R}\left(R^{n}\right) \\
& \cong \mathbb{M}_{n}(R) \\
& \cong D \otimes_{K} \mathbb{M}_{n}(A) \\
& \cong D \otimes_{K} \operatorname{End}_{A}\left(A^{n}\right) .
\end{aligned}
$$

Finally, if $e^{\prime} \in \operatorname{End}_{A}\left(A^{n}\right)$ is an idempotent endomorphism, then there is an idempotent $e \in \operatorname{End}_{R}\left(R^{n}\right)$ with

$$
\begin{aligned}
\operatorname{End}_{R}\left(D \otimes_{K} A^{n} e^{\prime}\right) & \cong \operatorname{End}_{R}\left(R^{n} e\right) \\
& \cong e \operatorname{End}_{R}\left(R^{n}\right) e \\
& \cong\left(1 \otimes e^{\prime}\right)\left(D \otimes_{K} \operatorname{End}_{A}\left(A^{n}\right)\right)\left(1 \otimes e^{\prime}\right) \\
& \cong D \otimes_{K} e^{\prime} \operatorname{End}_{A}\left(A^{n}\right) e^{\prime} \\
& \cong D \otimes_{K} \operatorname{End}_{A}\left(A^{n} e^{\prime}\right),
\end{aligned}
$$

as claimed.

For the remainder of this section, we will assume that $R$ is a basic indecomposable square-free ring with complete set $E=\left\{e_{1}, \ldots, e_{n}\right\}$ of orthogonal primitive idempotents. We define a binary relation $\leq$ on $E$ by

$$
e_{i} \leq e_{j} \quad \text { in case } \quad e_{i} R e_{j} \neq 0 .
$$


In general, this relation need not be either transitive or anti-symmetric.

For each $e_{i} \leq e_{j}$ in $E$, fix $0 \neq s_{i j} \in e_{i} R e_{j}$ with the proviso that $s_{i i}=e_{i}$ for each $i=1, \ldots, n$. Then the set

$$
B=\left\{s_{i j}: e_{i} \leq e_{j}\right\}
$$

is a basis for $R$ (over $E$ ). So if $B=\left\{s_{i j}\right\}$ is a basis for $R$ over $E$, then since $\operatorname{dim}_{e_{i} R e_{i}}\left(e_{i} R e_{j}\right) \leq 1$ for each $i, j$, we have that as additive groups,

$$
R=\sum_{i=1}^{n} \sum_{j=1}^{n} e_{i} R e_{j}=\sum_{e_{i} \leq e_{j}} e_{i} R e_{i} s_{i j} .
$$

If $B$ is a basis for $R$ over $E$, then we will usually abbreviate the isomorphisms $\varphi_{s_{i j}}$ by

for each $s_{i j} \in B$.

$$
\varphi_{i j}=\varphi_{s_{i j}}: e_{i} R e_{i} \longrightarrow e_{j} R e_{j}
$$

Let $B$ be a basis for $R$ over $E$ and let $B^{\prime} \subseteq B$ be a subset of $B$. We say that $B^{\prime}$ is solvable in case for each $i=1, \ldots, n$ there is an isomorphism $\mu_{i}: D \longrightarrow e_{i} R e_{i}$ such that

$$
\varphi_{i j}=\mu_{i}^{-1} \mu_{j}
$$

for all $s_{i j} \in B^{\prime}$. We will also then say that $\left(\mu_{i}\right)_{i=1}^{n}$ is a solution on $B^{\prime}$. In particular, the basis $B$ is a solvable basis for $R$ iff there is a solution $\left(\mu_{i}\right)_{i=1}^{n}$ on $B$. Note that if $\left(\mu_{i}\right)_{i=1}^{n}$ is a solution on $B^{\prime}$ and if $\alpha \in \operatorname{Aut} D$, then $\left(\alpha \mu_{i}\right)_{i=1}^{n}$ is also a solution on $B^{\prime}$.

Lemma 3.3. Let $B^{\prime} \subseteq B$ be solvable. For all $e_{i} \leq e_{j} \leq e_{k}$ in $E$ with $e_{i} \leq e_{k}$, if $s_{i j}, s_{j k}, s_{i k} \in B^{\prime}$, then there is a (necessarily unique) $d=d_{i j k} \in \operatorname{Cen}\left(e_{i} R e_{i}\right)$ with

$$
s_{i j} s_{j k}=d s_{i k} .
$$

Proof. Since $s_{i j} s_{j k} \in e_{i} R e_{i} s_{i k}$, there is a (necessarily unique) $d \in e_{i} R e_{i}$ with $s_{i j} s_{j k}=d s_{i k}$. We claim that $d \in \operatorname{Cen}\left(e_{i} R e_{i}\right)$. By hypothesis, there exist isomorphisms $\mu_{h}: D \longrightarrow e_{h} R e_{h}$ such that $\varphi_{g h}=\mu_{g}^{-1} \mu_{h}$ for all $s_{g h} \in B^{\prime}$. Thus, for each $d^{\prime} \in e_{i} R e_{i}$,

$$
\begin{aligned}
d^{\prime} d s_{i k} & =d^{\prime} s_{i j} s_{j k} \\
& =s_{i j} s_{j k}\left(d^{\prime}\right) \varphi_{i j} \varphi_{j k} \\
& =s_{i j} s_{j k}\left(d^{\prime}\right) \mu_{i}^{-1} \mu_{k} \\
& =d s_{i k}\left(d^{\prime}\right) \varphi_{i k} \\
& =d d^{\prime} s_{i k} .
\end{aligned}
$$

Thus, $d^{\prime} d=d d^{\prime}$ and $d \in \operatorname{Cen}\left(e_{i} R e_{i}\right)$, as claimed.

Corollary 3.4. Let $B^{\prime} \subseteq B$ be solvable, and let $e_{i} \leq e_{j} \leq e_{k}$ in $E$ with $e_{i} \leq e_{k}$. If $s_{i j}, s_{j k}, s_{i k} \in \overline{B^{\prime}}$, then $\varphi_{i k}=\varphi_{i j} \varphi_{j k}$.

Proof. Apply Lemmas 3.1 and 3.3. 
We now characterize square-free $D$-algebras in terms of solvable bases. For a different characterization, see Lemma 3.1 of [6].

Theorem 3.5. Let $R$ be a basic indecomposable square-free ring with division ring $D$. Then $R$ is a D-algebra if and only if $R$ has a solvable basis.

Proof. ( $\Longrightarrow$ ) Let $R=D \otimes_{K} A$ for some square-free $K$-algebra $A$ where $K=$ Cen $D$. We may assume that $E=\left\{1 \otimes e_{i}: e_{i} \in E_{A}\right\}$ is a complete set of orthogonal primitive idempotents for $R$ where $E_{A}=\left\{e_{1}, \ldots, e_{n}\right\}$ is a complete set of orthogonal primitive idempotents for $A$. We may choose a basis $B_{A}=\left\{s_{i j}: e_{i} \leq e_{j}\right\}$ for $A$ over $E_{A}$; then $B=\left\{1 \otimes s_{i j}: e_{i} \leq e_{j}\right\}$ is a basis for $R$ over $E$. For each $i$, let $\mu_{i}: d \longmapsto d \otimes e_{i}$. Then since $e_{i} A e_{i} \cong K$ for each $i$, each $\mu_{i}: D \longrightarrow\left(1 \otimes e_{i}\right) R\left(1 \otimes e_{i}\right)$ is an isomorphism. For each $s_{i j} \in B_{A}$ and $d \in D$,

$$
\left(d \otimes e_{i}\right)\left(1 \otimes s_{i j}\right)=\left(d \otimes e_{i} s_{i j}\right)=\left(d \otimes s_{i j} e_{j}\right)=\left(1 \otimes s_{i j}\right)\left(d \otimes e_{j}\right) .
$$

So $\left(d \otimes e_{i}\right) \varphi_{i j}=d \otimes e_{j}$, and $\left(\mu_{i}\right)_{i=1}^{n}$ is a solution for $B$. Thus, $B$ is a solvable basis for $R$.

$(\Longleftarrow)$ Let $E=\left\{e_{1}, \ldots, e_{n}\right\}$ be a complete set of orthogonal primitive idempotents for $R$ and let $B=\left\{s_{i j}: e_{i} \leq e_{j}\right\}$ be a solvable basis for $R$. Say $\left(\mu_{i}\right)_{i=1}^{n}$ is a solution for $B$. Define $\psi: D \longrightarrow R$ by

$$
(d) \psi=\sum_{i=1}^{n}(d) \mu_{i} .
$$

Observe that for all $e_{i} \leq e_{j}$ in $E$ and all $r \in R$,

$$
(d) \psi \cdot e_{i} r e_{j}=(d) \mu_{i} \cdot e_{i} r e_{j} \quad \text { and } \quad e_{i} r e_{j} \cdot(d) \psi=e_{i} r e_{j} \cdot(d) \mu_{j} .
$$

The map $\psi: D \longrightarrow R$ is clearly additive and since $(d) \mu_{i}\left(d^{\prime}\right) \mu_{j}=\delta_{i j}\left(d d^{\prime}\right) \mu_{i}$, where $\delta_{i j}$ is the Kronecker delta, it follows that $\psi$ is an isomorphism from $D$ onto a division subring $D^{\psi}$ of $R$.

We claim next that if $K=\operatorname{Cen} D$, then $K^{\psi}=(K) \psi$ is the center of $R$. First, we note that for each $i$, we have $(K) \mu_{i}=\operatorname{Cen}\left(e_{i} R e_{i}\right)$. Now suppose that $r \in \operatorname{Cen}(R)$. Then for each $i$, we have $e_{i} r=r e_{i}$, so

$$
r=e_{1} r e_{1}+\cdots+e_{n} r e_{n} .
$$

Clearly, $e_{i} r e_{i} \in \operatorname{Cen}\left(e_{i} R e_{i}\right)=(K) \mu_{i}$. Next, observe that if $e_{i} R e_{j} \neq 0$, then

$$
\left(e_{i} r e_{i}\right) s_{i j}=s_{i j}\left(e_{j} r e_{j}\right),
$$

so $e_{j} r e_{j}=\left(e_{i} r e_{i}\right) \varphi_{i j}$ or

$$
\left(e_{j} r e_{j}\right) \mu_{j}^{-1}=\left(e_{i} r e_{i}\right) \mu_{i}^{-1} \in K .
$$

Thus, there exists some unique $k \in K$ with $(k) \mu_{i}=e_{i} r e_{i}$ for all $i=1, \ldots, n$. That is, $r=(k) \psi \in K^{\psi}$. 
On the other hand, let $d \in K$. Then certainly, each $(d) \mu_{i} \in \operatorname{Cen}\left(e_{i} R e_{i}\right)$. So it will suffice to show that for each $i, j$ with $e_{i} R e_{j} \neq 0$, and for each $r \in R$,

$$
(d) \mu_{i} \cdot\left(e_{i} r e_{j}\right)=\left(e_{i} r e_{j}\right) \cdot(d) \mu_{j} .
$$

But if $e_{i} r e_{j} \neq 0$, then $e_{i} r e_{j}=e_{i} r^{\prime} e_{i} s_{i j}$ for some $e_{i} r^{\prime} e_{i} \in e_{i} R e_{i}$. Thus, since (d) $\mu_{i} \in \operatorname{Cen}\left(e_{i} R e_{i}\right)$,

$$
\begin{aligned}
(d) \mu_{i} \cdot\left(e_{i} r e_{j}\right) & =(d) \mu_{i} \cdot\left(e_{i} r^{\prime} e_{i} s_{i j}\right) \\
& =\left(e_{i} r^{\prime} e_{i}\right) \cdot(d) \mu_{i} \cdot s_{i j} \\
& =\left(e_{i} r^{\prime} e_{i}\right) s_{i j} \cdot(d) \mu_{i} \varphi_{i j} \\
& =\left(e_{i} r^{\prime} e_{i}\right) s_{i j} \cdot(d) \mu_{i} \mu_{i}^{-1} \mu_{j} \\
& =\left(e_{i} r e_{j}\right) \cdot(d) \mu_{j} .
\end{aligned}
$$

Thus, $\operatorname{Cen}(R)=K^{\psi}$ as claimed.

Now let $A$ be the $K^{\psi}$ subspace of $R$ spanned by $B$. By Lemma 3.3, for each $s_{i j}, s_{j k} \in B$, if $s_{i j} s_{j k} \neq 0$, then

$$
s_{i j} s_{j k}=d s_{i k}
$$

for some $d \in \operatorname{Cen}\left(e_{i} R e_{i}\right)$. But $\operatorname{Cen}\left(e_{i} R e_{i}\right)=(K) \mu_{i}$, so there is a (necessarily unique) $t \in K$ with $(t) \mu_{i}=d$. Then $(t) \psi \in \operatorname{Cen}(R)$ and

$$
s_{i j} s_{j k}=(t) \psi s_{i k} .
$$

Thus, $A$ is actually a $K^{\psi}$-subalgebra of $R$. In fact, it is square-free. Indeed, $E$ is a complete set of idempotents for $A$, and for each $e_{i}, e_{j}$, if $e_{i} A e_{j} \neq 0$, then $e_{i} A e_{j}$ is one dimensional over $K^{\psi}$ generated by $s_{i j}$.

Next, we claim that for each $a \in A$ and each $d \in D$,

$$
a(d) \psi=(d) \psi a .
$$

For this, it will clearly suffice to assume that $a=s_{i j}$ for some $e_{i} R e_{j} \neq 0$. But

$$
\begin{aligned}
((d) \psi)\left(s_{i j}\right) & =\left((d) \mu_{i}\right)\left(s_{i j}\right) \\
& =\left(s_{i j}\right)\left((d) \mu_{i} \varphi_{i j}\right) \\
& =\left(s_{i j}\right)\left((d) \mu_{j}\right) \\
& =\left(s_{i j}\right)((d) \psi),
\end{aligned}
$$

so $a(d) \psi=(d) \psi a$, as claimed.

Finally, the map $\tau: D \times A \longrightarrow R$ defined by

$$
\tau:(d, a) \longmapsto((d) \psi) a
$$

is clearly $K$-bilinear and universal, so there is the desired ring isomorphism $\bar{\tau}: D \otimes_{K} A \longrightarrow R$. 


\section{The main result.}

Let $R$ be a basic square-free ring with basic set $E=\left\{e_{1}, \ldots, e_{t}\right\}$ and with quiver $\mathcal{Q}=\mathcal{Q}(R)$. In this section we shall show that under certain conditions a solvable quiver will imply that $R$ is a $D$-algebra. Since we want the path structure of $\mathcal{Q}$ to be reflected in the algebra of $R$, we impose a restriction on $R$ that will provide the desired connection between $\mathcal{Q}$ and $R$.

If $\mathcal{E}$ is some set of vertices in $\mathcal{Q}$, then we say that $R$ is transitive on $\mathcal{E}$ in case for all $e, f, g \in \mathcal{E}$

$$
e J f J g=0 \Longrightarrow e J f=0 \text { or } f J g=0 .
$$

We say that $R$ is transitive if it is transitive on the set $E$ of all vertices of $\mathcal{Q}$ and locally transitive if it is transitive on the vertices of each cyclic component of $\mathcal{Q}$. If $R$ is transitive, then (see [6]) the relation $\leq$ on $E$, defined in Section 3, is a partial order, and $\mathcal{Q}$ is the Hasse diagram for this poset. For more on the property of transitivity for square-free rings see [6]. Much of the significance of transitivity for us is given in the next pair of lemmas.

Lemma 4.1. Let $R$ be transitive. For each pair $e_{i}, e_{j} \in E$, the following are equivalent:

(a) $e_{i} \leq e_{j}$;

(b) $e_{i} R e_{j} \neq 0$;

(c) There is a d-path from $e_{i}$ to $e_{j}$ in $\mathcal{Q}$.

In particular, $e_{i_{0}} \leq e_{i_{1}} \leq \cdots \leq e_{i_{k}}$ in $\mathcal{Q}$ iff there is a d-path in $\mathcal{Q}$ through the sequence $\left(e_{i_{0}}, e_{i_{1}}, \ldots, e_{i_{k}}\right)$.

Proof. The equivalence of (a) and (b) is by definition.

(b) $\Leftarrow(\mathrm{c})$. If there is an arrow from $e_{i}$ to $e_{k}$ and an arrow from $e_{k}$ to $e_{j}$, then $e_{i} J e_{k} \neq 0$ and $e_{k} J e_{j} \neq 0$, so by transitivity, $e_{i} J e_{k} J e_{j} \neq 0$. The conclusion follows by induction on the length of the path.

(b) $\Rightarrow$ (c). We may assume $e_{i} \neq e_{j}$. If $e_{i} R e_{j} \neq 0$, then $e_{i} J^{m} e_{j} \neq 0$ for some maximal $m \geq 1$. If $m=1$, then there is an arrow from $e_{i}$ to $e_{j}$. If $m>1$, then there is an $e_{k}$ with $e_{i} J^{m} e_{j} \supseteq e_{i} J e_{k} J^{m-1} e_{j} \neq 0$. So there is an arrow from $e_{i}$ to $e_{k}$ and, by induction, a $d$-path from $e_{k}$ to $e_{j}$.

Observe that if $R$ is transitive, then $\mathcal{Q}$ contains no directed cycles. Let

$$
B=\left\{s_{i j}: e_{i} \leq e_{j}\right\}
$$

be a basis for $R$ over $E$.

Lemma 4.2. If $R$ is transitive, and if there is a d-path in $\mathcal{Q}$ through the sequence $\left(e_{i_{0}}, e_{i_{1}}, \ldots, e_{i_{k}}\right)$ of vertices in $E$, then $s_{i_{0} i_{1}} \cdots s_{i_{k-1} i_{k}} \neq 0$. 
Proof. Suppose that $e_{1}, e_{2}, e_{3} \in E$ and that there is a $d$-path through the sequence $\left(e_{1}, e_{2}, e_{3}\right)$. We may assume that $e_{1}, e_{2}, e_{3}$ are distinct. Then by Lemma $4.1, e_{1} R e_{2} \neq 0$ and $e_{2} R e_{3} \neq 0$. So

$$
e_{1} J e_{2}=e_{1} R e_{2}=e_{1} R e_{1} s_{12} \neq 0,
$$

and similarly, $e_{2} J e_{3}=s_{23} e_{3} R e_{3} \neq 0$. Thus, by transitivity, $e_{1} J e_{2} J e_{3} \neq 0$, so that $s_{12} s_{23} \neq 0$. Now induct.

Lemma 4.3. If $R$ is transitive, and if $e_{i} \leq e_{j} \leq e_{k} \leq e_{\ell}$ in $E$, then there exists $v \in e_{j} R e_{k}$ with

$$
s_{i \ell}=s_{i j} v s_{k \ell} .
$$

Proof. This follows immediately from the fact that since $R$ is transitive and square-free, $e_{i} R e_{\ell}=e_{i} R e_{j} R e_{k} R e_{\ell}=s_{i j} e_{j} R e_{k} s_{k \ell}$. See [6].

Our next goal is to find sufficient conditions on $R$ for it to have a solvable basis. For this we first assume that $R$ is transitive and indecomposable. We will prove the main result for this case, extend that to stars of cyclic components, and then piece these together for the general result. A key part of that is a lemma that gives a condition under which we can extend solvability from a subquiver to a larger subquiver.

Let $\mathcal{Q}_{1}$ be a subquiver of $\mathcal{Q}$. If $B$ is a basis for $R$, then we set

$$
B\left(\mathcal{Q}_{1}\right)=\left\{s_{i j} \in B \text { : there is a } d \text {-path from } e_{i} \text { to } e_{j} \text { in } \mathcal{Q}_{1}\right\} .
$$

Let $V \subseteq E$. We denote by $V^{+}$the subquiver of $\mathcal{Q}$ whose arrows are precisely those that lie on some $d$-path of $\mathcal{Q}$ that originates at some $e \in V$. Similarly, we denote by $V^{-}$the subquiver whose arrows lie on some $d$-path of $\mathcal{Q}$ that terminates at some $e \in V$. So, the vertex set of $V^{+}$is the subposet of $\mathcal{Q}$ consisting of all upper bounds of the elements of $V$ and the vertex set of $V^{-}$ is the subposet of all lower bounds of the elements of $V$.

Next, we want to investigate some conditions under which a basis solvable on a subquiver can be modified to create a basis solvable on a larger subquiver.

Lemma 4.4. Let $R$ be an indecomposable square-free ring with quiver $\mathcal{Q}$, and suppose that $B$ is a basis for $R$. Let $\mathcal{Q}_{1}$ and $\mathcal{Q}_{2}$ be disjoint subquivers with both $B\left(\mathcal{Q}_{1}\right)$ and $B\left(\mathcal{Q}_{2}\right)$ solvable. If there is an arrow a from a vertex $e_{i}$ in $\mathcal{Q}_{1}$ to a vertex $e_{j}$ in $\mathcal{Q}_{2}$, then there is a basis $B^{\prime}$ with $B^{\prime}\left(\mathcal{Q}_{1} \cup\{a\} \cup \mathcal{Q}_{2}\right)$ solvable. In particular, if a subquiver $\mathcal{Q}^{\prime}$ of $\mathcal{Q}$ is a tree, then there is a basis $B^{\prime}$ for $R$ with $B^{\prime}\left(\mathcal{Q}^{\prime}\right)$ solvable.

Proof. Let $\left(\mu_{k}\right)_{k=1}^{n}$ be a sequence of isomorphisms $\mu_{k}: \longrightarrow e_{k} R e_{k}$ that form solutions on both $B\left(\mathcal{Q}_{1}\right)$ and $B\left(\mathcal{Q}_{2}\right)$. For each $e_{h} \leq e_{k}$ in $\mathcal{Q}$ let $s_{h k}^{\prime} \in e_{h} R e_{k}$ be given by

$$
s_{h k}^{\prime}= \begin{cases}s_{h j} s_{j k}, & \text { if } e_{h} \in \mathcal{Q}_{1} \text { and } e_{k} \in \mathcal{Q}_{2} \\ s_{h k}, & \text { otherwise. }\end{cases}
$$


Then $B^{\prime}=\left\{s_{h k}^{\prime}: e_{h} \leq e_{k}\right\}$ is a basis for $R$. Moreover, the sequence $\left(\nu_{k}\right)_{k=1}^{n}$ of isomorphisms given by

$$
\nu_{k}= \begin{cases}\mu_{j} \varphi_{s_{k j}^{\prime}}^{-1}, & \text { if } e_{k} \in \mathcal{Q}_{1} \text { and } e_{k} \leq e_{j} \\ \mu_{k}, & \text { otherwise }\end{cases}
$$

is a solution for $B^{\prime}\left(\mathcal{Q}_{1} \cup\{a\} \cup \mathcal{Q}_{2}\right)$. The final statement is now a simple induction.

Lemma 4.5. Let $R$ be a transitive square-free ring with quiver $\mathcal{Q}$ and basis B. Let $\mathcal{Q}_{1}$ be a connected subquiver of $\mathcal{Q}$, let a be an arrow of $\mathcal{Q}_{1}$, and let $\mathcal{Q}_{2}$ be the subquiver of $\mathcal{Q}_{1}$ obtained by deleting the arrow a. Assume that $B\left(\mathcal{Q}_{2}\right)$ is solvable. If a belongs to a bi-directed cycle of $\mathcal{Q}_{1}$, then there is a basis $B^{\prime}$ for $R$ with $B^{\prime}\left(\mathcal{Q}_{1}\right)$ solvable.

Proof. Let $\left(\mu_{i}\right)_{i=1}^{n}$ be a sequence of isomorphisms $\mu_{i}: D \longrightarrow e_{i} R e_{i}$ that form a solution for $B\left(\mathcal{Q}_{2}\right)$. Suppose that $a$ is an arrow from $e_{i}$ to $e_{j}$ and belongs to the bi-directed cycle $p \cup q$ where $p$ and $q$ are $d$-paths from $e_{\ell}$ to $e_{m}$. We may assume that $a$ is an arrow on $q$. By Lemma 4.3 there is a $v \in e_{i} R e_{j}$ with $s_{\ell m}=s_{\ell i} v s_{j m}$. Thus,

$$
\varphi_{v}=\varphi_{\ell i}^{-1} \varphi_{\ell m} \varphi_{j m}^{-1}=\mu_{i}^{-1} \mu_{j} .
$$

For each $e_{h} \leq e_{k}$ in $E$ define $s_{h k}^{\prime} \in e_{h} R e_{k}$ by

$$
s_{h k}^{\prime}= \begin{cases}s_{h i} v s_{j k}, & \text { if } e_{h} \leq e_{i} \leq e_{j} \leq e_{k} \\ s_{h k}, & \text { otherwise }\end{cases}
$$

Then $B^{\prime}=\left\{s_{h k}^{\prime}: e_{h} \leq e_{k}\right.$ in $\left.E\right\}$ is a basis for $R$ and $\left(\mu_{i}\right)_{i=1}^{n}$ is a solution for $B^{\prime}\left(\mathcal{Q}_{1}\right)$.

Let $X$ be an arbitrary quiver and let $\mathcal{V}$ be the $\mathbb{Z}_{2}$-space on the set $A$ of all arrows of $X$. Thus, we can also think of $\mathcal{V}$ as the Boolean algebra $\mathcal{P}(A)$ of all subsets of $A$. In the proof of the next lemma we will consider the $\mathbb{Z}_{2}$-subspace $\mathcal{B}(X)$ of $\mathcal{V}$ spanned by the bi-directed cycles of $X$.

Lemma 4.6. Let $H$ be a connected subquiver of $\mathcal{Q}$, let $u$ be a minimal element of $\mathcal{Q}$, let $w$ be a maximal element of $\mathcal{Q}$, let $p_{0}$ be a d-path from $u$ to $w$, and let $X$ be a subquiver of $u^{+} \cap w^{-}$such that $p_{0}$ lies in $X$ and every arrow in $X$ lies on some $d$-path in $X$ from $u$ to $w$. Suppose there is a basis $B$ for $R$ with $B\left(H \cup p_{0}\right)$ solvable. If $w \in H$ or if $u \in H$, then there is a basis $B^{\prime}$ for $R$ with $B^{\prime}(H \cup X)$ solvable.

Proof. We'll prove the case with $w \in H$; the other is dual. The proof will be by induction on $b=\operatorname{dim} \mathcal{B}(H \cup X)-\operatorname{dim} \mathcal{B}\left(H \cup p_{0}\right)$, the difference between the dimensions of the bi-directed cycle spaces of $H \cup X$ and $H \cup p_{0}$. So suppose that $b=0$. If there were some arrow $a$ in $X$ but not in $H \cup p_{0}$, then since there must be a $d$-path in $X$ from $u$ to $w$ through $a$, and since $a$ 
cannot be on $p_{0}$, there is some bi-directed cycle in $X$ containing $a$, contrary to $\operatorname{dim} \mathcal{B}(H \cup X)=\operatorname{dim} \mathcal{B}\left(H \cup p_{0}\right)$. So if $b=0$, then $H \cup X=H \cup p_{0}$, and we're done.

So assume that $b>0$. Then there must be some nontrivial $d$-path in $X$ no arrow of which is in $H \cup p_{0}$. Let $p_{1}$ be such a $d$-path of maximal length. Say the initial vertex of $p_{1}$ is $x$ and the terminal vertex is $y$. If there is a vertex of $p_{1}$ other than $y$ that is the initial vertex of a bi-directed cycle in $X$, let $x^{\prime}$ be the greatest such vertex. Otherwise let $x^{\prime}=x$. If there is a vertex different from $x^{\prime}$ on $p_{1}$ between $x^{\prime}$ and $y$ that is the terminal vertex of a bi-directed path in $X$, then let $y^{\prime}$ be the smallest such vertex. Otherwise, let $y^{\prime}=y$. Let $p$ be the sub $d$-path of $p_{1}$ from $x^{\prime}$ to $y^{\prime}$, and let $X^{\prime}=X \backslash p$. Then $p_{0}$ is in $X^{\prime}$ and every arrow in $X^{\prime} \backslash H$ lies on a $d$-path from $u$ to $w$ in $X^{\prime}$. But clearly, $\operatorname{dim} \mathcal{B}\left(H \cup X^{\prime}\right)<\operatorname{dim} \mathcal{B}(H \cup X)$, so by induction, there is a basis $B^{\prime}$ for $R$ with $B^{\prime}\left(H \cup X^{\prime}\right)$ solvable. Now every vertex of $p$ other than $x^{\prime}$ and $y^{\prime}$ has valence 2 in $H \cup X$. Thus, by Lemma 4.4, there is a basis $B^{\prime \prime}$ for $R$ with $B^{\prime \prime}(H \cup X)$ solvable.

Lemma 4.7. Let $R$ be an indecomposable transitive square-free ring with quiver $\mathcal{Q}$. If $\mathcal{Q}$ is solvable, then $R$ has a solvable basis.

Proof. Since $\mathcal{Q}$ is connected, there exist sequences $\left(u_{1}, \ldots, u_{n}\right)$ and $\left(w_{1}, \ldots\right.$, $w_{n}$ ) of minimal and maximal elements of $\mathcal{Q}$, with repetitions allowed, such that

$$
u_{i}, u_{i+1} \leq w_{i} \text { for all } i<n \text { and } u_{n} \leq w_{n}
$$

and $\mathcal{Q}=\left\{u_{1}, \ldots, u_{n}\right\}^{+} \cap\left\{w_{1}, \ldots, w_{n}\right\}^{-}$. We begin with some notation. For each $m$ let

$$
F_{m}=\left\{u_{1}, \ldots, u_{m}\right\}^{+} \cap\left\{w_{1}, \ldots, w_{m}\right\}^{-},
$$

and

$$
G_{m}=\left\{u_{1}, \ldots, u_{m}\right\}^{+} \cap\left\{w_{1}, \ldots, w_{m-1}\right\}^{-} .
$$

To prove this lemma, it suffices to show that for each $m$ :

(i) For $1 \leq m \leq n$, if $B$ is a basis for $R$ with $B\left(G_{m}\right)$ solvable, then there is a basis $B^{\prime}$ for $R$ with $B^{\prime}\left(F_{m}\right)$ solvable.

(ii) For $1 \leq m<n$, if $B$ is a basis for $R$ with $B\left(F_{m}\right)$ solvable, then there is a basis $B^{\prime}$ for $R$ with $B^{\prime}\left(G_{m+1}\right)$ solvable.

We will show (i); the arguments for (ii) are similar.

So let $m=1$. Then there must be a $d$-path $p_{0}$ from $u_{1}$ to $w_{1}$. By Lemma 4.4 there is a basis $B$ for $R$ with $B\left(p_{0}\right)$ solvable. Then by Lemma 4.6, there is a basis $B^{\prime}$ for $R$ with $B^{\prime}\left(u_{1}^{+} \cap w_{1}^{-}\right)$solvable. Next, let $1<m \leq n$ and assume that $B\left(G_{m}\right)$ is solvable. For $0 \leq k<m$, set

$$
H_{k}=\left(\left\{u_{m-k}, \ldots, u_{m}\right\}^{+} \cap w_{m}^{-}\right) \cup G_{m} .
$$

We will show by induction on $k$ that there is a basis $B^{\prime}$ for $R$ with $B^{\prime}\left(H_{k}\right)$ solvable. Let $k=0$. Then there is a $d$-path $p_{0}$ from $u_{m}$ to $w_{m}$, so by 
Lemmas 4.4 and 4.5 there is a basis $B^{\prime \prime}$ for $R$ with $B^{\prime \prime}\left(G_{m} \cup p_{0}\right)$ solvable. Thus, by Lemma 4.6 there is a basis $B^{\prime}$ for $R$ with $B^{\prime}\left(H_{0}\right)$ solvable. Now let $0 \leq k<m-1$ and assume there is a basis $B$ for $R$ with $B\left(H_{k}\right)$ solvable. If $u_{m-k-1} \in\left\{u_{m-k}, \ldots, u_{m}\right\}$ or if there is no $d$-path from $u_{m-k-1}$ to $w_{m}$, then $H_{k+1}=H_{k}$, and we're done. Otherwise, by hypothesis about the sequences $\left(u_{1}, \ldots, u_{n}\right)$ and $\left(w_{1}, \ldots, w_{n}\right)$, there must be sets $U^{\prime}=\left\{u_{m-k-1}=\right.$ $\left.u_{1}^{\prime}, u_{2}^{\prime}, \ldots, u_{h}^{\prime}\right\} \subseteq\left\{u_{m-k-1}, \ldots, u_{m}\right\}$ and $W^{\prime}=\left\{w_{1}^{\prime}, w_{2}^{\prime}, \ldots, w_{h}^{\prime}=w_{m}\right\} \subseteq$ $\left\{w_{m-k-1}, \ldots, w_{m}\right\}$ for which $U^{\prime}$ and $W^{\prime}$ form a weak $n$-crown. So since $\mathcal{Q}$ is solvable, Lemma 2.2 guarantees a pair of $d$-paths $p_{0}$ from $u_{1}^{\prime}$ to $w_{h}^{\prime}$ and $q$ from some $u_{i}^{\prime} \in U^{\prime} \backslash\left\{u_{1}^{\prime}\right\}$ to some $w_{j}^{\prime} \in W^{\prime} \backslash\left\{w_{h}^{\prime}\right\}$ that meet in some common vertex $v$. But $d$-paths from $u_{1}^{\prime}$ to $w_{j}^{\prime}$ and from $u_{i}^{\prime}$ to $w_{h}^{\prime}$ must be in $H_{k}$, so that $p_{0}$ is in $H_{k}$. In particular, $H_{k} \cup p_{0}=H_{k}$, and so by Lemma 4.6, there is a basis $B^{\prime}$ for $R$ such that $B^{\prime}\left(H_{k+1}\right)$ is solvable. By induction on $k$, then, there is a basis $B^{\prime}$ for $R$ with $B^{\prime}\left(H_{m-1}\right)$ solvable. But $H_{m-1}=F_{m}$, so we are done with (i).

Theorem 4.8. If $R$ is a locally transitive indecomposable square-free ring with solvable quiver, then $R$ has a solvable basis and hence is a D-algebra.

Proof. By Theorem 3.2 we may assume that $R$ is basic. By Lemma 2.3 we may assume that the cyclic components of the quiver $\mathcal{Q}$ of $R$ are labeled in a proper order $\left\{S_{1}, \ldots, S_{n}\right\}$. By Lemma 4.7 for each $i=1, \ldots, n$ there is a basis $B_{i}$ for $R$ with $B_{i}\left(S_{i}\right)$ solvable. For each tree $T_{j}$ in the subquiver of cut edges of $\mathcal{Q}$ there is a basis solvable on $T_{j}$. So by Lemma 4.4, for each $i=1, \ldots, n$, we may assume that $B_{i}\left(\operatorname{St}\left(S_{i}\right)\right)$ is solvable. Finally, use Lemma 4.4 and induction on $n$ to find a basis $B$ that is solvable on $\operatorname{St}\left(S_{1}\right) \cup$ $\operatorname{St}\left(S_{2}\right) \cup \cdots \cup \operatorname{St}\left(S_{n}\right)=\mathcal{Q}$. By Theorem $3.5, R$ is a $D$-algebra.

\section{Matrix representations.}

If $R=D \otimes_{K} A$ is a $D$-algebra, then $R$ acts essentially like the split $K$-algebra $A$. For example, a $D$-automorphism of $R$ is just a ring automorphism $\alpha$ of $R$ such that for some $K$-automorphism $\bar{\alpha}$ of $A$ (with $\alpha$ and $\bar{\alpha}$ now viewed as left operators)

$$
\alpha(d \otimes a)=d \otimes \bar{\alpha}(a)
$$

for all $d \in D$ and $a \in A$. Thus, the $D$-automorphism groups of $R$ are completely determined by the $K$-automorphism groups of $A$. For squarefree algebras these groups have been studied extensively. (See, for example, [2].) So if $R$ is a square-free ring satisfying the hypotheses of Theorem 4.8, then the entire analysis of the $K$-automorphism groups of the square-free algebra $A$ given in [2] translates to that of the $D$-automorphism groups of $R$.

In this section, however, we want to describe how the $D$-algebras $R$ that are locally transitive square-free rings with solvable quivers can be realized as twisted matrix rings. Thanks to Theorem 3.2 we can restrict ourselves to 
basic rings. The extension of these representations to the more general case is straightforward.

First, though, let $R=D \otimes_{K} A$ be an arbitrary basic square-free $D$ algebra. Then the $K$-algebra $A$ is split, basic, and square-free. Moreover, if $E=\left\{e_{1}, \ldots, e_{n}\right\}$ is a basic set of primitive idempotents for $A$, then $\left\{1 \otimes e_{1}, \ldots, 1 \otimes e_{n}\right\}$ is a basic set for $R$, and if $J$ is the Jacobson radical for $A$, then $D \otimes J$ is the Jacobson radical for $R$. In particular, both $A$ and $R$ have the same quiver $\mathcal{Q}$, and $R$ is transitive (locally transitive) iff $A$ is.

Now let $R=D \otimes_{K} A$ be a basic, locally transitive, indecomposable square-free ring with quiver $\mathcal{Q}$ and division ring $D$, and assume that $E=$ $\left\{e_{1}, \ldots, e_{n}\right\}$ is a basic set of primitive idempotents for $A$. Define a relation $\preceq$ on $E$ by

$$
e_{i} \preceq e_{j} \Longleftrightarrow \text { there is a } d \text {-path in } \mathcal{Q} \text { from } e_{i} \text { to } e_{j} .
$$

Let $e_{i}$ and $e_{j}$ belong to the same cyclic component of $\mathcal{Q}$. By Lemma 4.1, $e_{i} \preceq e_{j}$ iff $e_{i} A e_{j} \neq 0$. Thus, by Theorem 2.3 of [6], restricted to each cyclic component, this relation is a partial order with Hasse diagram the restriction of $\mathcal{Q}$ to that component. However, shrinking each cyclic component of $\mathcal{Q}$ to a point yields a tree in which there is at most one $d$-path from any one vertex to another. Thus, the relation $\preceq$ is a partial order on all of $E$ and the Hasse diagram of this poset, called the regular poset of $A$ and of $R$, is $\mathcal{Q}$. Therefore, we can assume that the labels for the elements of $E$ have been chosen so that $i \leq j$ if $e_{i} \preceq e_{j}$, for all $i, j$.

If $\Lambda$ is an arbitrary ring, then the $\Lambda$ incidence ring $\Lambda E$ of the finite poset $(E, \preceq)$ can be realized as the ring of all $n \times n$ matrices $\llbracket a_{i j} \rrbracket$ over $\Lambda$ with $a_{i j}=0$ whenever $e_{i} \npreceq e_{j}$. If $K$ is a field, then the $K$-incidence algebra $K E$ of $(E, \preceq)$ is a subalgebra of the algebra of all $n \times n$ upper triangular matrices over $K$. For each $1 \leq i, j \leq n$ let $e_{i j}$ be the $(i, j)$ matrix unit in $\mathbb{M}_{n}(K)$. Then the $K$-incidence algebra $K E$ has $K$-basis

$$
B(E)=\left\{e_{i j} \in \mathbb{M}_{n}(K) \mid e_{i} \preceq e_{j} \text { in } E\right\} .
$$

If $A$ is transitive with regular poset $E$, then in fact, $A$ is isomorphic to the subalgebra $K_{\xi} E$ of upper triangular matrices with $K$-basis $B(E)$ and with multiplication twisted by a two-dimensional cocycle $\xi$ of the poset $E$ with coefficients in $K^{*}$. (See [5], [1] Theorem 2.4, or [2] Theorem 1.14.)

More generally, suppose that $A$ is locally transitive. Consider the $K$-path algebra $K \mathcal{Q}$ of the quiver $\mathcal{Q}$ of $A$. By Gabriel's Theorem (see [8], Section 4; or [4] Theorem III.1.9) there is a surjective $K$-algebra homomorphism, called a Gabriel homomorphism for $A, \gamma: K \mathcal{Q} \longrightarrow A$ from $K \mathcal{Q}$ onto $A$ with $\gamma\left(e_{i}\right)=e_{i}$ for each $e_{i} \in E$. Now suppose that $p$ and $q$ are two $d$-paths in $\mathcal{Q}$ from $e$ to $f$ for which $p \cup q$ forms a bi-directed cycle in $\mathcal{Q}$. Then $p$ and $q$ must belong to the same cyclic component, and so since $A$ is locally transitive, 
by Lemma $4.2, e A f \neq 0$. Thus, $\operatorname{dim}_{K}(e A f)=1$ and so

$$
e A f=K \gamma(p)=K \gamma(q) \text {. }
$$

Thus, for each such pair $(p, q)$ of $d$-paths in $\mathcal{Q}$ there is a nonzero $\rho(p, q) \in K^{*}$ with

$$
\gamma(p)=\rho(p, q) \gamma(q)
$$

Next, let $I$ be the ideal

$$
I=\langle p-\rho(p, q) q: p \cup q \text { is a bidirected cycle in } \mathcal{Q}\rangle
$$

of the $K$-path algebra $K \mathcal{Q}$. Let $\bar{A}=K \mathcal{Q} / I$, and let $\bar{\gamma}: K \mathcal{Q} \longrightarrow \bar{A}$ be the natural surjective algebra homomorphism. Since $I \leq \operatorname{ker}(\gamma)$, it follows that $\gamma$ factors through $\bar{\gamma}$, say $\varphi: \bar{A} \longrightarrow A$ with $\gamma=\varphi \circ \bar{\gamma}$. Also, if $p$ and $q$ are two $d$-paths from $e$ to $f$, then $p$ and $q$ factor as

$$
p=p_{1} p_{2} \cdots p_{t} \quad \text { and } \quad q=q_{1} q_{2} \cdots q_{t},
$$

where for each $1 \leq k \leq t$, either $p_{k}=q_{k}$ or $p_{k} \cup q_{k}$ is a bi-directed cycle. Letting $\rho\left(p_{k}, q_{k}\right)=1$ if $p_{k}=q_{k}$, we can define $\rho(p, q) \in K^{*}$ by

$$
\rho(p, q)=\rho\left(p_{1}, q_{1}\right) \rho\left(p_{2}, q_{2}\right) \cdots \rho\left(p_{t}, q_{t}\right) \text {. }
$$

Then

$$
\gamma(p)=\rho(p, q) \gamma(q) .
$$

For each $e_{i} \in E$, let $\overline{e_{i}}=e_{i}+I \in \bar{A}$. Note that $\left\{\overline{e_{1}}, \ldots, \overline{e_{n}}\right\}$ is a basic set of primitive idempotents for $\bar{A}$. Suppose $e, f \in E$ with $\bar{e} \bar{A} \bar{f} \neq 0$. A routine check shows that $\operatorname{dim}_{K}(\bar{e} \bar{A} \bar{f})=1$ and thus, $\bar{A}$ is square-free. If $e \neq f$ and $e(K Q) f \neq 0$, then there is a path from $e$ to $f$ in $\mathcal{Q}$. Since $I$ contains no directed paths, we can conclude that $\bar{A}$ is transitive.

Thus, $\bar{A}$ is a transitive square-free $K$-algebra and hence $\bar{A} \cong K_{\xi} E$ for some two-dimensional cocycle $\xi$ of $E$. Combining these observations with Theorem 4.8, we have the following matrix realization of locally transitive square-free rings with solvable quivers:

Theorem 5.1. Let $R$ be a basic, locally transitive, indecomposable squarefree ring with solvable quiver $\mathcal{Q}$ on the basic set $E=\left\{e_{1}, \ldots, e_{n}\right\}$, and with division ring $D$. The relation defined on $E$ by

$$
e_{i} \preceq e_{j} \Longleftrightarrow \text { there is a d-path in } \mathcal{Q} \text { from } e_{i} \text { to } e_{j}
$$

is a partial order and the Hasse diagram of the poset $(E, \preceq)$ is isomorphic to the digraph $\mathcal{Q}$. Then there is a two-dimensional cocycle $\xi$ of the poset $E$ with coefficients in $K=\operatorname{Cen} D$ such that $R$ is isomorphic to a factor ring of the $D$ incidence ring $D_{\xi} E=D \otimes_{K}\left(K_{\xi} E\right)$ of $E$ twisted by $\xi$. Finally, $R$ is transitive iff $R$ is isomorphic to the twisted $D$-incidence ring $D_{\xi} E$. 
Acknowledgements. The authors would like to thank Jessica K. Sklar for several helpful discussions about and comments on earlier versions of this work.

\section{References}

[1] F.W. Anderson, Transitive square-free algebras, J. Algebra, 62 (1980), 61-85, MR 81f:16031, Zbl 0442.16012.

[2] F.W. Anderson and B.K. D'Ambrosia, Square-free algebras and their automorphism groups, Comm. Alg., 24 (1996), 3163-3191, MR 98a:16027, Zbl 0883.16015.

[3] F.W. Anderson and K.R. Fuller, Rings and Categories of Modules (2d ed.), SpringerVerlag, New York-Berlin-Heidelberg, 1992, MR 94i:16001, Zbl 0765.16001.

[4] M. Auslander, I. Reiten and S.O. Smalø, Representation Theory of Artin Algebras, Cambridge University Press, Cambridge-New York-Melbourne, 1995, MR 96c:16015, Zbl 0834.16001.

[5] W.E. Clark, Cohomology of semigroups via topology - with an application to semigroup algebras, Comm. Algebra, 4 (1976), 979-997, MR 54 \#2764, Zbl 0342.20037.

[6] B.K. D'Ambrosia, Square-free rings, Comm. Algebra, 5 (1999), 2045-2071, MR 2000h:16033, Zbl 0922.16013.

[7] K.R. Fuller and J.K. Haack, Rings with quivers that are trees, Pacific J. Math., 76 (1978), 371-379, MR 58 \#16764, Zbl 0389.16011.

[8] P. Gabriel, Auslander-Reiten sequences and representation-finite algebras, Lecture Notes in Math., 831, Springer-Verlag, New York-Berlin-Heidelberg, 1980, 1-70, MR 82i:16030, Zbl 0445.16023.

Received June 13, 2001 and revised April 7, 2003.

UNIVERSITY OF OREGON

Eugene, OR 97403

E-mail address: anderson@math.uoregon.edu

John CARRoll University

University Heights, OH 44118

E-mail address: bdambrosia@jcu.edu 